Chirurg 2020 $91: 977$

https://doi.org/10.1007/s00104-020-01226-3

Online publiziert: 23. Juni 2020

(c) Springer Medizin Verlag GmbH, ein Teil von Springer Nature 2020

\section{Originalpublikation}

Chen QY, Xie JW, Zhong Q, Wang JB et al (2020) Safety and efficacy of indocyanine green tracer-guided lymph node dissection during laparoscopic radical gastrectomy in patients with gastric cancer. JAMA Surgery 155(4):300-311. https://doi.org/10.1001/ jamasurg.2019.6033.

Hintergrund und Fragestellung. Die radikale Lymphadenektomie (LAD) wird nach der S3-Leitlinie „Magenkarzinom“ als integraler Bestandteil der onkologischen Magenresektion empfohlen. Im Rahmen der laparoskopischen Gastrektomie werden in zunehmenden Maße Verfahren entwickelt, die intraoperativ eine metastasenfokussierte Lymphknotendissektion in Echtzeit ermöglichen. Eines dieser Verfahren basiert auf der intravenösen/peritumoralen Applikation des Tracers Indocyaningrün (ICG), welcher mittels „near-infrared“ (NIR) Fluoreszenztechnologie in Lymphknoten (LK) intraoperativ visualisiert werden kann. Inwieweit mit dieser semiquantitativen Technik die onkologisch notwendige LAD beim Magenkarzinom gesteuert und verbessert werden kann, ist Gegenstand aktueller Diskussionen. Zur Frage der Machbarkeit und Sicherheit dieser Methode wurde jetzt von einer chinesischen Arbeitsgruppe eine prospektiv randomisierte Studie publiziert.

Methoden. Insgesamt wurden 258 Patienten mit Magenkarzinom (cT1-cT4a) in die Untersuchung eingeschlossen, alle Patienten wurden primär operiert (114 laparoskopische subtotale Magenresektionen und 144 laparoskopische Gastrektomien). Bei 129 Patienten wurde am Tag

W. Schröder · H. Fuchs · C. J. Bruns

Klinik für Allgemein-, Viszeral- und Tumorchirurgie, Universitätsklinik Köln, Köln, Deutschland

\title{
Indocyaningrün(ICG)-gesteuerte Lymphadenektomie bei der laparoskopischen Gastrektomie
}

vor der Operation endoskopisch ICG peritumoral injiziert und die intraoperative LAD ICG-gesteuert durchgeführt. Bei 129 Patienten erfolgte eine konventionelle D2-LAD (Non-ICG-Gruppe). Als primärer Outcome-Parameter wurde die Gesamtzahl der resezierten LK definiert.

Ergebnisse. Die Gesamtzahl der resezierten LK war in der ICG-Gruppe signifikant höher als in der Non-ICG-Gruppe (50,5 vs. 42,0 LK, $p<0,01)$. Dieser Befund konnte sowohl für die perigastrischen als auch die LK des D2-Kompartiments (extragastrische LK) nachgewiesen werden und war unabhängig vom Resektionsverfahren (Gastrektomie vs. subtotale Resektion). Die durchschnittliche Anzahl der resezierten LK-Metastasen war für beide Gruppen bei vergleichbaren Tumorstadien mit 5,6 und 5,7 LK vergleichbar. Die diagnostische Sensitivität der ICG-Fluoreszenz wurde mit 56,3\% (410 von 728 LK) und die Spezifität mit 46,1\% (2669 von 5785 LK) berechnet. Die postoperativen Komplikationsraten und deren Schweregrad waren für beide Gruppen gleich.

Fazit. Die aktuelle Studie ist aufgrund des Studiendesigns und der Studiendurchführung von hoher Qualität und zeigt das mögliche Potenzial der komplikationslos durchführbaren ICG-gesteuerten Lymphknotendissektion beim Magenkarzinom. Kritisch anzumerken bleibt die hohe Rate falsch negativer ICG-Messungen, die möglicherweise dadurch zu erklären ist, dass die Fluoreszenztechnologie eine histopathologisch bestehende Mikrometastasierung nicht nachweisen kann. Die Bedeutung dieses
Verfahrens liegt eher in der Detektion pathologischer LK außerhalb des geplanten nodalen Resektionsausmaßes. Inwieweit das initiale Tumorstadium und auch neoadjuvante Therapiekonzepte Einfluss auf den Lymphabfluss und damit die ICG-Messung haben, wird mit dieser Studie auch nicht beantwortet. Gegenwärtig hat die standardisierte Durchführung einer D2-LAD beim Magenkarzinom mit sorgfältiger histopathologischer Aufarbeitung des Resektates weiterhin Bestand.

\section{Korrespondenzadresse}

Prof. Dr. W. Schröder, FACS, FEBS

Klinik für Allgemein-, Viszeral- und Tumorchirurgie, Universitätsklinik Köln Kerpener Str. 62, 50937 Köln, Deutschland wolfgang.schroeder@uni-koeln.de

Interessenkonflikt. W. Schröder, H. Fuchs und C.J. Bruns geben an, dass kein Interessenkonflikt besteht. 